\title{
COMPACT DIRECTED SPACES $\left({ }^{1}\right)$
}

\author{
BY \\ L. E. WARD, JR.
}

\begin{abstract}
A directed space is a partially ordered topological space in which each two elements have a common predecessor. It is a consequence of a theorem of A. D. Wallace that a compact directed space is acyclic if each of its principal ideals is acyclic. This result is extended by considering the situation where at most finitely many principal ideals are not acyclic. It turns out that some of the elements which generate nonacyclic principal ideals must be maximal and that the $p$ th cohomology group of the space must contain the $p$ th cohomology group of such a principal ideal as a direct summand. In the concluding sections it is shown that these spaces can be made acyclic by dividing out a closed ideal which contains all of the nonacyclic principal ideals, and some results on the acyclicity properties of minimal partial orders on compact spaces are proved.
\end{abstract}

1. Introduction. If $(X, \Gamma)$ is a partially ordered set and $A \subset X$ we write

$$
\Gamma A=\{x \in X:(x, a) \in \Gamma \text { for some } a \in A\}
$$

and $A \Gamma$ is defined dually. If $x$ and $y$ are elements of $X$ we regard $x \leqq y,(x, y) \in \Gamma$, $x \in \Gamma y$ and $y \in x \Gamma$ as synonyms. As usual, $x<y$ means that $x \leqq y$ and $x \neq y$. A set $A=\Gamma A$ is an ideal of the partially ordered set, and if $x \in X$ then $\Gamma x$ is a principal ideal.

If $X$ is a topological space and the partial order $\Gamma$ is a closed subset of $X \times X$, then $(X, \Gamma)$ is a partially ordered space. A directed space is a partially ordered space $(X, \Gamma)$ in which $\Gamma x \cap \Gamma y$ is nonempty for all elements $x$ and $y$ of $X$.

We say that a topological space is acyclic if it has the SWAK (=AlexanderSpanier) groups of a point [3]. A partially ordered space is pointwise acyclic if each of its principal ideals is acyclic.

In the setting of directed spaces Wallace's acyclicity theorem [6], [7] asserts that a compact directed space is acyclic if it is pointwise acyclic. We note that the proof given in [7] applies to an independent but related theorem for an appropriate class of topological semigroups, rather than to partially ordered spaces. However it admits a straightforward extension to directed spaces which we include in the present paper (Theorem 3.1).

Presented to the Society, January 26,1969 under the title On the cohomology of compact directed spaces; received by the editors September 23, 1969.

AMS 1969 subject classifications. Primary 5455, 5456; Secondary 0620, 2205.

Key words and phrases. Directed space, partially ordered space, acyclic space.

(1) This research was supported by a grant from the National Science Foundation.

Copyright (C) 1970, American Mathematical Society 
The chief purpose of this paper is to explore the extent to which the cohomology of a compact directed space is determined by the cohomology of its principal ideals. Among other results we see that essentially complete information is obtained if only finitely many elements of the space generate nonacyclic principal ideals. This is followed by a theorem which asserts that such spaces can always be made acyclic by the operation of forming an appropriate quotient space; specifically the quotient space formed by "dividing out" a closed ideal which contains all of the nonacyclic principal ideals. In the concluding section there are some results on the acyclicity properties of minimal partial orders on a compact space.

2. Preliminary results. In this section we summarize most of the lemmas required in the proofs to follow. Proofs of Propositions 2.1 and 2.2 can be found in [8].

Proposition 2.1. The principal ideals of a partially ordered space are closed sets.

An element $x$ of a partially ordered set $(X, \Gamma)$ is termed maximal (minimal) if $(x, y) \in \Gamma$ implies $x=y$ (if $(y, x) \in \Gamma$ implies $x=y$ ). It is convenient to let $\operatorname{Max} X$ denote the set of maximal elements of $X$ and $\operatorname{Min} X$ denote the set of minimal elements of $X$.

Proposition 2.2. If $(X, \Gamma)$ is a compact partially ordered space, then Max $X$ and $\operatorname{Min} X$ are nonempty.

A zero of a partially ordered set $(X, \Gamma)$ is an element 0 such that $\{0\} \times X \subset \Gamma$. Dually, a unit is an element 1 such that $X \times\{1\} \subset \Gamma$. Zeroes and units, when they exist, are necessarily unique.

Proposition 2.3. A compact partially ordered space is a directed space if and only if it contains a zero.

Proof. Let $(X, \Gamma)$ be a compact partially ordered space. If $X$ has a zero, then it is obvious that $\Gamma x \cap \Gamma y$ is nonempty for all elements $x$ and $y$ of $X$. Conversely, if the space is directed, then by Proposition 2.1 the principal ideals are a family of closed sets with the finite intersection property. Since $X$ is compact the principal ideals have nonempty intersection, and thus a zero exists.

Proposition 2.4. If $F$ is a closed subset of the compact partially ordered space $(X, \Gamma)$, then $\Gamma F$ is a closed set.

Proof. Choquet [1] or Ward [9].

Proposition 2.5. Let $(X, \Gamma)$ be a compact partially ordered space and let $P$ and $Q$ be closed subsets of $X$ such that $P$ and $\Gamma Q$ are disjoint. Then there exists an open set $U$ such that $P \subset U=U \Gamma$ and $Q \subset X-\bar{U}$.

Proof. For each $q \in Q$ the sets $P \times\{q\}$ and $\Gamma$ are disjoint and hence $q$ has a compact neighborhood $K_{q}$ such that $P \times K_{q}$ and $\Gamma$ are disjoint. Since $Q$ is compact 
there is a compact neighborhood $K$ of $Q$ such that $P \times K$ and $\Gamma$ are disjoint, and by Proposition 2.4 the set $\Gamma K$ is closed and hence $U=X-\Gamma K$ is open. It is clear that $P \subset U$ and $Q \subset X-\bar{U}$.

Proposition 2.6. Let $(X, \Gamma)$ be a compact partially ordered space and let $P$ and $Q$ be closed subsets of $X$ such that $P$ and $\Gamma Q$ are disjoint and $Q \subset \operatorname{Max} X$. Then $X$ contains closed proper ideals $M$ and $N$ such that $X=M \cup N, P \subset M-N$ and $Q \subset N-M$.

Proof. By Proposition 2.5 there is an open set $U$ such that $P \subset U=U \Gamma$ and $Q \subset X-\bar{U}$, so we let $N=X-U$ and $M=\Gamma(\bar{U})$. It is obvious that $P \subset M-N$, $Q \subset N-\bar{U}$ and $X=M \cup N$. Moreover, because $Q \subset \operatorname{Max} X$, it follows that $Q \subset N-M$.

Proposition 2.7. Let $(X, \Gamma)$ be a compact connected directed space and let $C=\{p \in X: \Gamma p$ is not connected $\}$. If $C$ is nowhere dense then $C$ is empty.

Proof. If $x \in X$ let $K_{x}$ denote the component of $\Gamma x$ which contains $x$. We claim that $0 \in K_{x}$. For otherwise $\Gamma x$ is contained in the union of disjoint open sets $U$ and $V$ with $K_{x} \subset U$ and $0 \in V$. Since $\Gamma$ is closed there is an open neighborhood $W$ of $x$ such that $\Gamma t \subset U \cup V$ for each $t \in W$. Since $C$ is nowhere dense there exists $t \in(W-C) \cap U$, and since $\Gamma t$ is connected it is contained in $U$. But $0 \in \Gamma t-U$, a contradiction. Now suppose $y \in X$ and $z \in \Gamma y$. Since $0 \in K_{y} \cap K_{z}$ and $K_{z} \subset \Gamma y$ it follows that $z \in K_{y}$ and hence $\Gamma y=K_{y}$.

On occasion we shall require certain standard results concerning the cohomology groups of a compact space, pair or triad. Specifically, we assume the exactness of the cohomology sequence of a pair and of the Mayer-Vietoris sequence of a compact triad [3, p. 29]. We shall also have occasion to use the strong excision theorem [3, p. 89]. The three propositions below were formulated and proved by A. D. Wallace [5].

Proposition 2.8 (Extension Theorem). If $X$ is a compact Hausdorff space, $A$ is a closed subset of $X$ and $e \in H^{p}(A)$, then there is an open set $N$ which contains $A$ such that e can be extended to $H^{p}(\bar{N})$, i.e. e lies in the image of the natural homomorphism $H^{p}(\bar{N}) \rightarrow H^{p}(A)$.

Proposition 2.9 (Reduction TheOREM). If $X$ is a compact Hausdorff space, $A$ is a closed subset of $X$ and $e \in H^{p}(X)$ such that $e \mid A=0$, i.e. e lies in the kernel of the natural homomorphism $H^{p}(X) \rightarrow H^{p}(A)$, then there exists an open set $N$ which contains $A$ such that $e \mid \bar{N}=0$.

Proposition 2.10 (MAP EXCISION TheOReM). Let $X$ and $Y$ be compact Hausdorff spaces and suppose that $A$ and $B$ are closed subsets of $X$ and $Y$, respectively. If $f$ is $a$ map of $X$ onto $Y$ which carries $X-A$ topologically onto $Y-B$, then $f$ induces an isomorphism of $H^{p}(Y, B)$ onto $H^{p}(X, A)$. 
Now suppose $(X, \Gamma)$ is a partially ordered space, $A$ is a closed ideal of $X$ and $e \in H^{p}(A)$. A $\Gamma$-roof for $e$ is a closed ideal $R$ of $X$ which is minimal relative to (i) $A \subset R$ and (ii) $e$ cannot be extended to $H^{p}(R)$. If $h \in H^{p}(X)$ then a $\Gamma$-floor for $h$ is a closed ideal $F$ of $X$ which is minimal relative to $h \mid F \neq 0$. In case $\Gamma$ is the discrete partial order $((x, y) \in \Gamma$ if and only if $x=y)$ then $R$ and $F$ are usually termed a roof and floor, respectively, and in this situation the two existence theorems below are well known (see, for example, [2]). Their proofs generalize readily to the present setting, and we sketch them briefly.

Proposition 2.11. If $(X, \Gamma)$ is a compact partially ordered space, $A$ is a closed ideal of $X$ and $e \in H^{p}(A)$ such that $e$ cannot be extended to $H^{p}(X)$, then $e$ has a $\Gamma$-roof.

Proof. Let $\mathscr{M}$ be a maximal nest of closed ideals which contain $A$ and to which $e$ cannot be extended. Then $M_{0}=\bigcap \mathscr{M}$ is a closed ideal containing $A$. That $M_{0}$ is a $\Gamma$-roof for $e$ follows from the extension theorem and the fact that any neighborhood of $M_{0}$ contains a member of $\mathscr{M}$.

Proposition 2.12. If $(X, \Gamma)$ is a compact partially ordered space, if $0 \neq h \in H^{p}(X)$ and if $K$ is a closed ideal of $X$ such that $h \mid K=0$, then $h$ has $a \Gamma$-floor which contains $K$.

Proof. Let $\mathscr{M}$ be a maximal nest of closed ideals such that $M \supset K$ and $h \mid M \neq 0$ for each $M \in \mathscr{M}$. Then $M_{0}=\bigcap \mathscr{M}$ is a closed ideal containing $K$. That $M_{0}$ is a $\Gamma$-floor for $h$ follows from the reduction theorem and the fact that any neighborhood of $M_{0}$ contains a member of $\mathscr{M}$.

We conclude this section with two more lemmas of an entirely technical nature which will prove useful in the sequel.

Proposition 2.13. Let $X$ be a compact Hausdorff space, $K$ a closed subset of $X$ and suppose $h \in H^{p}(K)$ such that $h \neq 0$ and $X$ is a roof for $h$. If $S$ and $T$ are closed proper subsets of $X$ such that $X=S \cup T$ and $K \subset S$, then $S \cap T$ is not acyclic.

Proof. If, on the contrary, $S \cap T$ is acyclic then by the Mayer-Vietoris sequence for the triple $(X, S, T)$ we have

$$
H^{p}(X) \approx H^{p}(S) \oplus H^{p}(T),
$$

and since $h$ is extendable to $H^{p}(S)$ it follows that $X$ is not a roof for $h$.

Proposition 2.14. Let $(Y, \Gamma)$ be a compact directed space with unit $y_{1}$ and let $F$ be a closed subset of $Y$ such that $y_{1} \notin F$. If $a \Gamma=\left\{x, y_{1}\right\}$ for each $x \in F$, then $Y$ contains a closed ideal $K$ and $a$ closed subset $M$ such that $F \subset K^{0}-M, Y=K \cup M$ and $K \cap M$ is an ideal.

Proof. By Proposition 2.5 there exists an open set $U$ such that $y_{1} \in U=U \Gamma$ and $F \subset Y-\bar{U}$. Let $K=Y-U$ and define $M=\bar{U} \cup \Gamma(\bar{U} \cap K)$. The only conclusions of the proposition which are not immediate are the assertions that $F$ and $M$ are dis- 
joint and that $M \cap K$ is an ideal. If there exists $x \in F \cap M$, then $x \in \Gamma(\bar{U} \cap K)$ and hence $x<t$ where $t \in \bar{U} \cap K$. But then $t \neq y_{1}$, contrary to the hypothesis that $x \Gamma=\left\{x, y_{1}\right\}$. Since $K$ is an ideal, a simple computation shows that

$$
\begin{aligned}
\Gamma(M \cap K) & =\Gamma(M) \cap \Gamma(K)=\Gamma(\bar{U}) \cap \Gamma(K) \\
& =\Gamma(\bar{U}) \cap K=(\bar{U} \cap K) \cup \Gamma(\bar{U} \cap K)=M \cap K .
\end{aligned}
$$

3. Some structure theorems for directed spaces. We begin with a proof of Wallace's acyclicity theorem for directed spaces.

THEOREM 3.1. If $(X, \Gamma)$ is a pointwise acyclic compact directed space and if $K$ is a closed subset of $X$, then $\Gamma K$ is acyclic.

Proof. Since $\Gamma x$ is acyclic for each $x \in K$, each $\Gamma x$ is connected. By Proposition 2.3 there is a zero in $X$ and hence $\Gamma K$ is the union of connected sets with a point in common. Therefore $\Gamma K$ is connected.

Now suppose there is a least integer $n>0$ such that for some closed set $K \subset X$ it follows that $H^{n}(\Gamma K) \neq 0$. Let $0 \neq h \in H^{n}(\Gamma K)$; by the floor theorem (Proposition 2.12) $h$ has a $\Gamma$-floor $F$. Thus $F$ is not acyclic and hence is not a principal ideal. By Proposition 2.6, $F$ contains closed proper ideals $M$ and $N$ such that $F=M \cup N$. Thus $h|M=0=h| N$. Now consider the Mayer-Vietoris sequence

$$
H^{n-1}(M \cap N) \stackrel{\Delta}{\longrightarrow} H^{n}(F) \stackrel{J^{*}}{\longrightarrow} H^{n}(M) \oplus H^{n}(N) .
$$

If $n=1$ then $\Delta=0$, and if $n>1$ then the induction hypothesis assures us that $H^{n-1}(M \cap N)=0$ and hence $\Delta=0$. In any event $J^{*}$ is injective so that $J^{*}(h \mid F)$ $=(h|M, h| N) \neq 0$, a contradiction.

COROLlary 3.2. Suppose the $n$-sphere $S^{n}$ is partially ordered as a directed space and that each principal ideal is acyclic in dimensions $0,1, \ldots, n-1$. Then $S^{n}$ contains a unit.

Proof. For if card $\left(\operatorname{Max} S^{n}\right)>1$ then every principal ideal is homeomorphic to a subcontinuum of $R^{n}$ and is therefore acyclic (in all dimensions). But then by Theorem 3.1, $S^{n}$ is acyclic, which is a contradiction.

It follows easily from the exact sequence of a pair that if $A$ is a closed acyclic subset of the space $X$ then $H^{p}(X, A) \approx H^{p}(X)$ for all $p>0$. Combining this with Theorem 3.1 we obtain

Corollary 3.3. If $(X, \Gamma)$ is a pointwise acyclic compact directed space, and if $K$ and $Y$ are closed ideals of $X$ with $Y \subset K$, then $K$ is acyclic and $H^{p}(K, Y)=0$ for all $p>0$.

There is one more corollary we state here which will have frequent applications.

COROLlaRY 3.4. If $(X, \Gamma)$ is a compact directed space and if $X$ contains closed sets $X_{1}$ and $X_{2}$ such that $X=X_{1} \cup X_{2}$ and $X_{1} \cap X_{2}$ is a pointwise acyclic ideal, then $H^{p}(X) \approx H^{p}\left(X_{1}\right) \oplus H^{p}\left(X_{2}\right)$ for all $p>0$. 
Proof. Consider the Mayer-Vietoris sequence

$$
H^{p-1}\left(X_{1} \cap X_{2}\right) \stackrel{\Delta}{\longrightarrow} H^{p}(X) \stackrel{J^{*}}{\longrightarrow} H^{p}\left(X_{1}\right) \oplus H^{p}\left(X_{2}\right) \stackrel{I^{*}}{\longrightarrow} H^{p}\left(X_{1} \cap X_{2}\right) .
$$

Since $X_{1} \cap X_{2}$ is pointwise acyclic, it is acyclic by Theorem 3.1. Consequently $J^{*}$ is bijective.

For the remainder of this section we consider the case where $(X, \Gamma)$ is a compact directed space with finitely many nonacyclic principal ideals. The next two theorems establish the initial step of a fairly complicated induction.

THEOREM 3.5. Let $(X, \Gamma)$ be a compact directed space and suppose there exists exactly one element $x_{1}$ of $X$ such that $\Gamma x_{1}$ is not acyclic. Then $x_{1} \in \operatorname{Max} X$.

Proof. First suppose $\Gamma x_{1}$ is not connected. If $x_{1} \in X-\operatorname{Max} X$, then $X=$ $\bigcup\{\Gamma x: x \in \operatorname{Max} X\}$ is the union of connected sets with zero in common, and hence $X$ is connected. But then by Proposition 2.7 the principal ideal $\Gamma x_{1}$ is connected, a contradiction, so that $x_{1} \in \operatorname{Max} X$.

Now suppose $\Gamma x_{1}$ is connected so that $H^{n}\left(\Gamma x_{1}\right) \neq 0$ for some $n>0$. Suppose $0 \neq h \in H^{n}\left(\Gamma x_{1}\right)$ and that $x_{1}<x_{2} \in X$. Then, relative to the space $\Gamma x_{2}, h$ has a $\Gamma$-roof $R$.

Suppose $x_{1} \in \operatorname{Max} R$. Now $h$ does not extend to $R$ so that $R \neq \Gamma x_{1}$, i.e. $\operatorname{Max} R$ contains elements other than $x_{1}$. By Proposition 2.6, $R=R_{1} \cup R_{2}$ where $R_{1}$ and $R_{2}$ are closed proper ideals of $R$ and $x_{1} \in R_{1}-R_{2}$. Thus $R_{2}$, and hence $R_{1} \cap R_{2}$, are pointwise acyclic and hence acyclic, by Theorem 3.1. However by Proposition 2.13, $R_{1} \cap R_{2}$ is not acyclic.

Consequently $x_{1} \in R-\operatorname{Max} R$ and hence $x_{1}<y \in \operatorname{Max} R$. Since $\Gamma y$ is acyclic it is clear that $\Gamma y=R$. Moreover there is no element $z$ such that $x_{1}<z<y$, for otherwise $h$ would extend to $H^{n}(\Gamma z)$, contrary to the acyclicity of $\Gamma z$. Thus the hypotheses of Proposition 2.14 are satisfied so that $\Gamma y$ contains a closed proper ideal $K$ and a closed set $M$ such that $x_{1} \in K^{0}-M$ (the interior is taken relative to the space $\Gamma y$ ), $\Gamma y=K \cup M$ and $K \cap M$ is an ideal. Moreover, $K \cap M$ is a pointwise acyclic ideal so that by Corollary $3.4, H^{n}(\Gamma y) \approx H^{n}(K) \oplus H^{n}(M)$. But $H^{n}(\Gamma y)=0$ and $h$ extends to $H^{n}(K)$. This is a contradiction and therefore $x_{1} \in \operatorname{Max} X$.

THEOREM 3.6. Let $(X, \Gamma)$ be a compact directed space and suppose there exists exactly one element $x_{1}$ of $X$ such that $\Gamma x_{1}$ is not acyclic. Then $H^{n}(X) \approx H^{n}\left(\Gamma x_{1}\right)$ for all $n=0,1, \ldots$

Proof. By Theorem 3.5, $x_{1} \in \operatorname{Max} X$. If $\Gamma x_{1}$ is not connected, then the fact that $\Gamma t$ is connected for all $t \in X-\left\{x_{1}\right\}$ establishes the fact that $X-\left\{x_{1}\right\}$ is connected. By Proposition 2.7, $X$ is not connected, and hence $x_{1}$ is an isolated point of $X$. Thus $H^{0}(X) \approx H^{0}\left(\Gamma x_{1}\right)=G \oplus G$, where $G$ is the coefficient group. However, if $\Gamma x_{1}$ is connected then $X=\bigcup\{\Gamma t: t \in X\}$ is also connected, so that $H^{0}(X) \approx$ $H^{0}\left(\Gamma x_{1}\right)=G$. 
Now suppose there exists $n>0$ such that $H^{n}\left(\Gamma x_{1}\right)$ and $H^{n}(X)$ are not isomorphic; then the injection $j: \Gamma x_{1} \rightarrow X$ induces a homomorphism $j^{*}: H^{n}(X) \rightarrow H^{n}\left(\Gamma x_{1}\right)$ which is either not surmorphic or not injective.

If $j^{*}$ is not surmorphic then there exists $h \in H^{n}\left(\Gamma x_{1}\right)$ which does not extend to $H^{n}(X)$, and hence $h$ has a $\Gamma$-roof; without loss of generality we assume that $X$ is that $\Gamma$-roof. Then Max $X$ contains elements other than $x_{1}$, so that by Proposition 2.6, $X$ is the union of closed proper ideals $X_{1}$ and $X_{2}$ with $x_{1} \in X_{1}-X_{2}$. Thus $X_{2}$ is pointwise acyclic, so that by Theorem 3.1, $X_{1} \cap X_{2}$ is acyclic. But by Proposition 2.13 the contrary is true.

On the other hand, if $j^{*}$ is surmorphic but not injective then there exists $e \in H^{n}(X)$ such that $e \neq 0$ and $j^{*}(e)=0$. Then $e$ has a $\Gamma$-floor $F$ which contains $\Gamma x_{1}$ properly, and therefore Max $F$ contains elements other than $x_{1}$. By Proposition 2.6, $F$ is the union of closed proper ideals $F_{1}$ and $F_{2}$ with $x_{1} \in F_{1}-F_{2}$. Therefore $F_{1} \cap F_{2}$ is a pointwise acyclic ideal and we may apply Corollary 3.4. That is, $H^{n}(F) \approx H^{n}\left(F_{1}\right) \oplus H^{n}\left(F_{2}\right)$ where the isomorphism (see the proof of Corollary 3.4) is the natural one: $J^{*}(e)=\left(e\left|F_{1}, e\right| F_{2}\right)$. But this is a contradiction, for the fact that $F$ is a floor for $e$ implies that $e \mid F_{1}=0$, and $e \mid F_{2}=0$ since $F_{2}$ is pointwise acyclic and hence acyclic.

In the statements of the next two theorems note that the case $n=1$ is encompassed by Theorems 3.5 and 3.6.

THEOREM 3.7. Let $(X, \Gamma)$ be a compact directed space and suppose $X$ contains exactly $n$ elements which generate nonacyclic principal ideals. Then those $n$ elements can be labeled $x_{1}, \ldots, x_{n}$ in such a way that for some $m, 1 \leqq m \leqq n,\left\{x_{m}, x_{m+1}, \ldots, x_{n}\right\}$ $\subset \operatorname{Max} X$, and if $1 \leqq i<m$, then there exists $j \geqq m$ such that $x_{i}<x_{j}$. Finally, if $x_{p}<x<x_{q}$, then $x \in\left\{x_{1}, \ldots, x_{n}\right\}$.

THEOREM 3.8. With the hypotheses and notation of Theorem 3.7, $H^{p}(X)$ contains all of the groups $H^{p}\left(\Gamma x_{m}\right), \ldots, H^{p}\left(\Gamma x_{n}\right)$ as direct summands, with the isomorphism induced by the injection map.

Suppose $n>1$ and that both Theorem 3.7 and Theorem 3.8 have been established for the case of fewer than $n$ elements generating nonacyclic principal ideals.

First, assume that $\Gamma x_{p}$ and $\Gamma x_{q}$ are not acyclic and that $x_{p}<x<x_{q}$. Then $\Gamma x$ is a compact directed space which contains a positive number (less than $n$ ) of elements which generate nonacyclic principal ideals. By the induction hypothesis one of those elements must be maximal in $\Gamma x$, i.e. $x$ generates a nonacyclic principal ideal.

We enumerate the $n$ elements which generate nonacyclic principal ideals arbitrarily: $x_{1}, \ldots, x_{n}$. Suppose none of these elements is maximal. Then there exists $y \in \operatorname{Max} X$ with $x_{1}<y$. Since $\Gamma y$ is acyclic it follows from the induction hypothesis that $\Gamma y$ contains all of the elements $x_{1}, \ldots, x_{n}$, and by a straightforward application of Proposition 2.2 we may assume that $y$ is minimal with respect to that property. Since $\Gamma y$ is connected, it is a space satisfying the hypotheses of Proposition 2.7 and 
hence $\Gamma x_{1}, \ldots, \Gamma x_{n}$ are all connected. At this point it is helpful to divide the argument into two cases.

Case 1. No two distinct elements of $\left\{x_{1}, \ldots, x_{n}\right\}$ are comparable.

By the minimality of $y$ it follows that $x_{i} \Gamma \cap \Gamma y=\left\{x_{i}, y\right\}$ for each $i=1, \ldots, n$. If we write $F=\Gamma x_{1} \cup \cdots \cup \Gamma x_{n-1}$, then $F \cap \Gamma x_{n}$ is pointwise acyclic, and therefore by Corollary 3.4

$$
H^{p}\left(\bigcup_{i=1}^{n} \Gamma x_{i}\right) \approx H^{p}(F) \oplus H^{p}\left(\Gamma x_{n}\right)
$$

for all $p>0$. Since $H^{p}\left(\Gamma x_{n}\right) \neq 0$ for some $p>0$, there exists $h \in H^{p}\left(\bigcup_{i=1}^{n} \Gamma x_{i}\right)$, $h \neq 0$, and in the space $\Gamma y, h$ has a $\Gamma$-roof $R$. If $R \neq \Gamma y$ it follows that $\left\{x_{1}, \ldots, x_{n}\right\}$ $\subset \operatorname{Max} R$, but since $h$ does not extend to $H^{p}(R)$ we infer that $\operatorname{Max} R-\left\{x_{1}, \ldots, x_{n}\right\}$ is nonempty. By Proposition 2.6, $R$ is the union of closed proper ideals $S$ and $T$, with $\left\{x_{1}, \ldots, x_{n}\right\} \subset S-T$. But then $S \cap T$ is pointwise acyclic and hence acyclic, and this contradicts Proposition 2.13. Consequently $R=\Gamma y$ and we can apply Proposition 2.14 to the space $\Gamma y$. That is, $\Gamma y$ contains a closed ideal $K$ and a closed set $M$ such that $\left\{x_{2}, \ldots, x_{n}\right\} \subset K^{0}-M, \Gamma y=K \cup M$ and $K \cap M$ is a pointwise acyclic ideal. By Corollary 3.4, $H^{p}(\Gamma y) \approx H^{p}(K) \oplus H^{p}(M)$ and by the induction hypothesis $H^{p}(K)$ is a nontrivial group. This contradicts the acyclicity of $\Gamma y$.

Case 2. The elements of $\left\{x_{1}, \ldots, x_{n}\right\}$ can be labeled so that $x_{1}<\cdots<x_{k}$ forms a maximal chain, for some $k>1$.

For this situation we require a variation of Proposition 2.14 which depends on Theorem 3.5. By Proposition 2.5 there exists an open set $U$ of the space $\Gamma y$ such that $\left\{x_{2}, \ldots, x_{n}, y\right\} \subset U=U \Gamma$ and $x_{1} \in \Gamma y-\bar{U}$. Let $K=\Gamma y-U$ and define $M=$ $\bar{U} \cup \Gamma(\bar{U} \cap K)$. Obviously $K$ is an ideal, $x_{1} \in K^{0}$ (interior relative to the space $\Gamma y$ ) and $K \cup M=\Gamma y$. If $x_{1} \in M$ then it is clear that $x_{1} \in \Gamma(\bar{U} \cap K)$ and hence $x_{1}<t$ for some $t \in \bar{U}-U$. But such an element $t$ generates an acyclic principal ideal $\Gamma t$, and $\Gamma t$ has exactly one member which does not generate an acyclic principal ideal. This contradicts Theorem 3.5 and hence $x_{1} \in K^{0}-M$. As in the proof of Proposition 2.14, $K \cap M$ is an ideal, and it is clearly pointwise acyclic. Therefore, by Corollary 3.4, $H^{p}(\Gamma y) \approx H^{p}(K) \oplus H^{p}(M)$ for all $p>0$, and in particular $H^{p}(K)=0$ for all $p>0$. But $K$ contains exactly one element $x_{1}$ such that $H^{p}\left(\Gamma x_{1}\right) \neq 0$ for some $p>0$, and by Theorem 3.6, $H^{p}(K) \approx H^{p}\left(\Gamma x_{1}\right)$.

Consequently some members of $\left\{x_{1}, \ldots, x_{n}\right\}$ lie in Max $X$. In order to complete the proof of Theorem 3.7 it remains to show that if $\left\{x_{m}, \ldots, x_{n}\right\} \subset \operatorname{Max} X$ and $\left\{x_{1}, \ldots, x_{m-1}\right\} \subset X-\operatorname{Max} X$, and if $i \leqq m-1$ then $x_{i}<x_{j}$ for some $j \geqq m$. If not then we may assume $x_{i}$ is maximal relative to this property, and by Proposition 2.5 there is an open set $U$ such that $\left\{x_{m}, \ldots, x_{n}\right\} \subset U=U \Gamma$ and $x_{i} \in X-\bar{U}$. Applying the induction hypothesis to the directed space $X-U$, it follows that $x_{i} \in \operatorname{Max}(X-U)$. If $x_{i}<x \in \operatorname{Max} X-\left\{x_{m}, \ldots, x_{n}\right\}$ then $\Gamma x$ is acyclic so that by Theorem 3.1 the set $\Gamma x_{i}$ is acyclic, a contradiction. Therefore $x_{i} \in \operatorname{Max} X$, which contradicts the hypothesis. 
To prove Theorem 3.8 we consider first the case $p=0$. It must be shown that each component of $\Gamma x_{i}$ lies in a distinct component of $X$. We let $K_{0}$ denote the component of $X$ which contains 0 . If $x \in X-K_{0}$ then $\Gamma x$ is not connected and hence $X-K_{0}$ consists of finitely many isolated points. Therefore, if $x_{i} \in\left\{x_{1}, \ldots, x_{n}\right\}$ then the components of $\Gamma x_{i}$ are $\Gamma x_{i} \cap K_{0}$ and a subfamily of $X-K_{0}$.

Now let $p>0$ and suppose $\left\{x_{1}, \ldots, x_{n}\right\}$ has $x_{n}$ for a unit. Then either $X=\Gamma x_{n}$ (in which case the theorem is trivial) or Max $X-\left\{x_{n}\right\}$ is nonempty and consists of elements which generate acyclic principal ideals. The argument we use at this point is patterned after the proof of Theorem 3.6. If $j$ denotes the inclusion $j: \Gamma x_{n} \rightarrow X$ we will show that $j^{*}$ is bijective. For if $j^{*}$ is not surmorphic there exists $h \in H^{p}\left(\Gamma x_{n}\right)$ which does not extend to $H^{p}(X)$, and so $h$ has a $\Gamma$-roof $R$ which properly contains $\Gamma x_{n}$. By Proposition 2.6, $R$ is the union of proper closed ideals $R_{1}$ and $R_{2}$, with $x_{n} \in R_{1}-R_{2}$. Moreover, no member of $\left\{x_{1}, \ldots, x_{n}\right\}$ lies in $R_{2}$, for none of them is maximal in $R_{2}$ (Theorem 3.7). Thus $R_{2}$ and $R_{1} \cap R_{2}$ are pointwise acyclic and hence acyclic. By Corollary 3.4 we have

$$
H^{p}(R) \approx H^{p}\left(R_{1}\right) \oplus H^{p}\left(R_{2}\right) \approx H^{p}\left(R_{1}\right)
$$

from which it follows that since $h$ extends to $H^{p}\left(R_{1}\right)$, it must also extend to $H^{p}(R)$. This contradicts the fact that $R$ is a $\Gamma$-roof for $h$.

On the other hand, if $j^{*}$ is surmorphic but not injective, then there exists $e \in H^{p}(X)$ such that $e \neq 0$ and $j^{*}(e)=0$. Then $e$ has a $\Gamma$-floor $F$ which contains $\Gamma x_{n}$ properly. Thus $F$ has maximal elements other than $x_{n}$ and hence $F$ can be represented as the union of closed proper ideals, $F_{1}$ and $F_{2}$, with $x_{n} \in F_{1}$ and all maximal elements of $F_{2}$ generate acyclic principal ideals. It follows that $F_{2}$, and hence $F_{1} \cap F_{2}$, are acyclic and therefore $H^{p}(F) \approx H^{p}\left(F_{1}\right)$. But then $e \mid F_{1} \neq 0$, contrary to the fact that $F$ is a floor for $e$.

Now suppose $x_{n-1}$ and $x_{n}$ are distinct elements of $\operatorname{Max} X$. We complete the proof of Theorem 3.8 with a variant of the proof of Proposition 2.14. By Proposition 2.5 there exists an open set $U$ such that $x_{n} \in U=U \Gamma$ and $\left\{x_{1}, \ldots, x_{n-1}\right\} \subset X-\bar{U}$. By the induction hypothesis $H^{p}(X-U)$ contains $H^{p}\left(\Gamma x_{n-1}\right)$ as a direct summand. Further, since $\Gamma t$ is acyclic for each $t \in \bar{U}-U$, it follows from Theorems 3.1 and 3.7 that $\Gamma(\bar{U}-U)$ is acyclic. Since

$$
\begin{aligned}
X & =(X-U) \cup(\bar{U} \cup \Gamma(\bar{U}-U)), \\
\Gamma(\bar{U}-U) & =(X-U) \cap(\bar{U} \cup \Gamma(\bar{U}-U)),
\end{aligned}
$$

it follows from Corollary 3.4 that

$$
H^{p}(X) \approx H^{p}(\bar{U} \cup \Gamma(\bar{U}-U)) \oplus H^{p}(X-U)
$$

and hence $H^{p}(X)$ contains $H^{p}\left(\Gamma x_{n-1}\right)$ as a direct summand.

For the case $n=2$ it is possible to state a slightly stronger result.

THEOREM 3.9. Let $X$ be a compact directed space with exactly two elements, $x_{1}$ and $x_{2}$, which generate nonacyclic principal ideals. Then at least one of those elements 
lies in $\operatorname{Max} X$. If $x_{2} \in \operatorname{Max} X$ then either $x_{1}<x_{2}$ or $x_{1} \in \operatorname{Max} X$. If $x_{1}<x_{2}$ then $H^{p}(X) \approx H^{p}\left(\Gamma x_{2}\right)$ for all $p=0,1, \ldots$ If $\left\{x_{1}, x_{2}\right\} \subset \operatorname{Max} X$ then $H^{p}(X) \approx H^{p}\left(\Gamma x_{1}\right)$ $\oplus H^{p}\left(\Gamma x_{2}\right)$ for all $p=0,1, \ldots$.

The stronger result does not hold if $X$ has as many as three elements generar: $\mathrm{ng}$ nonacyclic principal ideals. In the Euclidean 3-space let $X$ consist of the bounidry of the square $\left\{\left(a_{1}, a_{2}, 0\right): 0 \leqq a_{1}, a_{2} \leqq 1\right\}$, together with the line segment joining $(0,0,0)$ to $(2,1,1)$ and the line segment joining $(0,0,0)$ to $(1,2,1)$. Let $X$ have the coordinatewise partial order, i.e. $\left(a_{1}, a_{2}, a_{3}\right) \leqq\left(b_{1}, b_{2}, b_{3}\right)$ if and only if $a_{1} \leqq b_{i}$ for $i=1,2,3$. Then $X$ is a compact directed space and there are exactly three points of $X$ which generate nonacyclic principal ideals: $x=(1,1,0), y=(2,1,1)$ and $z=$ $(1,2,1)$. Moreover, $\operatorname{Max} X=\{y, z\}$ and $H^{1}(\Gamma y) \approx H^{1}(\Gamma z) \approx H^{1}(X) \neq 0$.

4. A result on quotient spaces. In this section we establish the fact that any compact directed space with at most finitely many nonacyclic principal ideals can be made acyclic via the operation of identifying an appropriate closed ideal with a point.

If $(X, \Gamma)$ is a partially ordered space and $F$ is a closed ideal of $X$, let $X / F$ denote the quotient space induced by the equivalence relation which identifies elements of $F$, and let $\rho: X \rightarrow X / F$ be the projection map. Define a relation $\Gamma_{F}$ on $X / F$ by $(\rho(x), \rho(y)) \in \Gamma_{F}$ if and only if $(x, y) \in \Gamma$.

The following proposition is straightforward (compare with Lemma 3.1 of [9]).

Proposition 4.1. If $(X, \Gamma)$ is a compact partially ordered space and $F$ is a closed ideal of $X$ then $\left(X / F, \Gamma_{F}\right)$ is a compact partially ordered space, and if $x \in X$ then $\Gamma_{F} \rho(x)$ and $\Gamma x / F \cap \Gamma x$ are order homeomorphic. If, in addition, $(X, \Gamma)$ is a directed space, then so is $\left(X / F, \Gamma_{F}\right)$, and $\{F\}=\rho(0)$ is the zero of $X / F$.

Proposition 4.2. Let $(X, \Gamma)$ be a compact directed space and suppose $F$ is a closed ideal of $X$ which contains all the elements $x \in X$ such that $\Gamma x$ is not connected. If $y \in X-F$ then $\Gamma y \cap F$ is connected and $(X / F)$ is connected.

Proof. To see that $\Gamma y \cap F$ is connected, suppose the contrary; then $\Gamma y \cap F$ is the union of nonempty disjoint closed sets $A$ and $B$ with $0 \in B$. Let $U$ and $V$ be disjoint open sets such that $A \subset U$ and $B \subset V$. Since $\Gamma y$ is connected, there exists a net $y_{\alpha}$ in $(\Gamma y \cap U)-A$ such that $\lim y_{\alpha}=z \in A$. Note that $y_{\alpha} \notin F$ and hence $\Gamma y_{\alpha}$ is connected. Moreover $\Gamma y_{\alpha}$ meets both $U$ and $V$ since $0 \in B \subset V$, and hence $\Gamma y_{\alpha}-$ $(U \cup V)$ is not empty. Since $\Gamma$ is closed, the set $\Gamma z-(U \cup V)$ is not empty. But this is a contradiction since $\Gamma z \subset \Gamma y \cap F \subset A \cup B$.

To prove that $X / F$ is connected we observe that this is trivial if $X=F$, and if $X-F$ is not empty, then $X / F=\bigcup\left\{\Gamma_{F} \rho(y): y \in X-F\right\}$. Since each $\Gamma_{F} \rho(y)$ is the image under $\rho$ of the connected set $\Gamma y$, and since each of these sets contains $\rho(F)$, it follows that $X / F$ is connected. 
THEOREM 4.3. Let $X$ be a compact directed space with only finitely many nonacyclic principal ideals, all of which lie in the closed ideal $F$. If $y \in X-F$ then $\Gamma y \cap F$ is acyclic.

Proof. That $\Gamma y \cap F$ is acyclic in dimension 0 has been proved in Proposition 4.2. Now suppose $H^{p}(\Gamma y \cap F) \neq 0$ for some $p>0$. We may assume that $y_{0}$ is the infimum of a chain $C \subset X-F$, which is maximal with respect to the property that $H^{p}(\Gamma y \cap F)$ $\neq 0$ for each $y \in C$. By Theorem 3.8 there exists $x \in \operatorname{Max}(\Gamma y \cap F)$ such that $H^{p}(\Gamma x) \neq 0$ and (since there are only finitely many such elements) there exists such an $x$ in $\Gamma y_{0} \cap F$. If $y_{0} \in F$ then $\Gamma y_{0}=\Gamma y_{0} \cap F$ is not acyclic, and it follows from the Extension Theorem that $\Gamma y$ is not acyclic for some $y \in C$. This is a contradiction and consequently $y_{0} \in X-F$. By Proposition 2.5 there is an open set $U=U \Gamma$ such that $y_{0} \in U$ and $F \cap \bar{U}$ is empty. Since $\Gamma t$ is acyclic for each $t \in \partial U \cap \Gamma y_{0}$, it follows that $\Gamma\left(\partial U \cap \Gamma y_{0}\right)$ is acyclic (Theorems 3.1 and 3.7). By the Mayer-Vietoris sequence for the triple $\left(\Gamma y_{0}, \Gamma y_{0}-U, \Gamma y_{0} \cap(\bar{U} \cup \Gamma(\partial U))\right.$ :

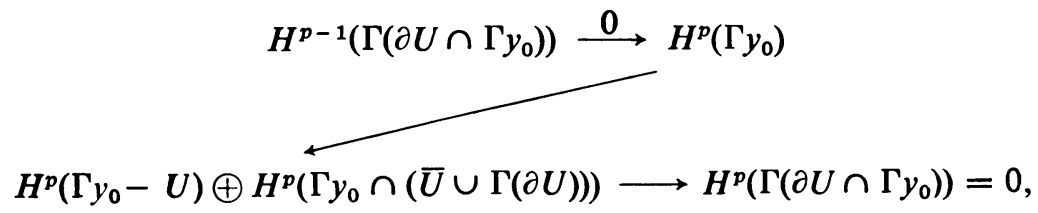

it follows that

$$
H^{p}\left(\Gamma y_{0}\right) \approx H^{p}\left(\Gamma y_{0}-U\right) \oplus H^{p}\left(\Gamma y_{0} \cap(\bar{U} \cup \Gamma(\partial U))\right)
$$

and hence $H^{p}\left(\Gamma y_{0}-U\right)=0$. But $H^{p}\left(\Gamma y_{0}-U\right)$ contains $H^{p}(\Gamma x)$ as a direct summand, by Theorem 3.8, and this is a contradiction.

COROLlaRY 4.4. If $X$ is a compact directed space with only finitely many nonacyclic principal ideals, all of which lie in the closed ideal $F$, then $X / F$ is acyclic.

Proof. By Theorem 3.1 it is enough to show that $\Gamma_{F} \rho(y)$ is acyclic for each $y \in X-F$, and hence it suffices to show that $\Gamma y / \Gamma y \cap F$ is acyclic for each $y \in X-F$. By Theorem 4.3 and the exact sequence for the pair $(\Gamma y, \Gamma y \cap F)$ it follows that $H^{p}(\Gamma y, \Gamma y \cap F) \approx H^{p}(\Gamma y)$ for all $p=0,1, \ldots$, and by the Map Excision Theorem (Proposition 2.10) we have $H^{p}(\Gamma y / \Gamma y \cap F) \approx H^{p}(\Gamma y, \Gamma y \cap F)$. Since $\Gamma y$ is acyclic by hypothesis, the theorem is proved.

As an example of an application of this result we can prove the following.

COROLLARY 4.5. If the $n$-cell $B^{n}$ is partially ordered as a directed space so that the bounding (n-1)-sphere $S^{n-1}$ is an ideal, then either $S^{n-1}$ contains infinitely many nonacyclic principal ideals or there exists $x \in B^{n}-S^{n-1}$ with $\Gamma x$ nonacyclic.

For certain lower dimensional examples it is not hard to see that Corollary 4.4 is true without the hypothesis that only finitely many principal ideals are nonacyclic. It would be interesting to know if this is true in general, thus permitting a stronger conclusion in Corollary 4.5 . 
5. Some remarks on minimal partial orders. We begin this section by recalling a theorem from [9].

THEOREM 5.1. If $(X, \Gamma)$ is a compact directed space with connected principal ideals, then $X$ admits a partial order which is minimal with respect to those properties.

This result was of interest since it was crucial to the proof of Koch's arc theorem [4] given in that paper. We note here that Theorem 5.1 is merely the 0 -dimensional analogue of a much stronger result.

THEOREM 5.2. If $(X, \Gamma)$ is a pointwise acyclic, compact directed space, then $X$ admits a partial order which is minimal with respect to those properties.

Proof. Let $\Gamma=\bigcap \Gamma_{\alpha}$ where $\Gamma_{\alpha}$ is a nest of partial orders satisfying

(i) $\Gamma_{\alpha}$ is closed,

(ii) $\{0\} \times X \subset \Gamma_{\alpha}$,

(iii) $\Gamma_{\alpha} x$ is acyclic, for each $x \in X$.

It is a simple exercise to verify that $\Gamma$ is a closed partial order and that $(X, \Gamma)$ is directed. Since $\Gamma x=\bigcap \Gamma_{\alpha} x$, it follows that each principal $\Gamma$-ideal is connected. It remains to show that $H^{n}(\Gamma x)=0$ for each $x \in X$ and each $n>0$. If, on the other hand, there exists $x \in X$ and $n>0$ such that $H^{n}(\Gamma x)$ contains $h \neq 0$, then by the Extension Theorem there is an open set $V \supset \Gamma x$ such that $h$ can be extended to $H^{n}(\bar{V})$. But there exists an $\alpha$ such that $\Gamma x \subset \Gamma_{\alpha} x \subset V$ and hence $h$ can be extended to $H^{n}\left(\Gamma_{\alpha} x\right)$, a contradiction.

We now consider a related question.

THEOREM 5.3. Let $X$ be a compact space and suppose $\Gamma$ is a partial order on $X$ which is minimal with respect to $(X, \Gamma)$ being a directed space with connected principal ideals. Then each principal dual ideal of $(X, \Gamma)$ is connected.

Proof. If some $x \Gamma$ is not connected then $x \Gamma=P \cup Q$ where $P$ and $Q$ are nonempty disjoint closed sets and $x \in P$. By Proposition 2.2 there exists $q \in \operatorname{Min} Q$ and $p \in \operatorname{Max}(\Gamma q \cap P)$, so that $p \Gamma \cap \Gamma q=\{p, q\}$. We may now exploit an argument first used in [9]. Since $X$ is a Hausdorff space, there are disjoint open sets $U$ and $V$ with $p \in U$ and $q \in V$. Let

$$
F=\{(x, y) \in X \times X: x \Gamma \cap \Gamma y-(U \cup V) \neq 0\} .
$$

Because $\Gamma$ is closed, so is $F$, and hence

$$
\Delta=\Gamma-(U \times V-F)
$$

is also closed. Since $\Gamma$ is reflexive and $U$ and $V$ are disjoint, one sees that $\Delta$ is reflexive, and the asymmetry of $\Gamma$ implies that $\Delta$ has the same property. To see that $\Delta$ is transitive, suppose that $a \Delta b$ and $b \Delta c$ but $(a, c) \in X \times X-\Delta$. Since $(a, c) \in \Gamma$, it is clear that

$$
(a, c) \in U \times V-F
$$


and thus $a \Gamma \cap \Gamma c \subset U \cup V$, so that $b \in U$ or $b \in V$. If $b \in U$ then, since $c \in V$ and $(b, c) \in \Delta$, we infer that $(b, c) \in F$ and consequently

$$
b \Gamma \cap \Gamma c-(U \cup V) \neq 0 .
$$

But then

$$
a \Gamma \cap \Gamma c-(U \cup V) \neq 0,
$$

i.e. $(a, c) \in F$, a contradiction. A similar contradiction ensues if $b \in V$ and therefore $\Delta$ is transitive.

Now let $y \in X$; if $y \in X-V$ then $\Delta y=\Gamma y$ and hence $\Delta y$ is connected. If $y \in V$ then for each $x \in \Delta y-V$, the set $\Delta x=\Gamma x$ is connected and contains the zero element, and hence

$$
\Delta y-V=\bigcup\{\Delta x: x \in \Delta y-V\}
$$

is connected. Since $\Gamma y$ is connected, each component of $\Gamma y \cap \bar{V}$ meets $\Delta y-V$; but $\Gamma y \cap \bar{V}=\Delta y \cap \bar{V}$ so that $\Delta y$ is connected.

We have shown that $(X, \Delta)$ is a directed space with connected principal ideals. Moreover, $\Delta$ is properly contained in $\Gamma$, because $(p, q) \in \Gamma-\Delta$. This contradicts the minimality of $\Gamma$, and the theorem follows.

It has been pointed out to me by Professor E. D. Tymchatyn that Theorem 5.3 is no longer true if the word "connected" is replaced by the word "acyclic". Nevertheless it would still be interesting to know if there are reasonable conditions under which an extension of this sort is possible.

\section{REFERENCES}

1. G. Choquet, Convergences, Ann. Univ. Grenoble Sect. Sci. Math. Phys. 23 (1948), 57-112. MR 10, 53.

2. Haskell Cohen, A cohomological definition of dimension for locally compact Hausdorff spaces, Duke Math. J. 21 (1954), 209-224. MR 16, 609.

3. S. T. Hu, Cohomology theory, Markham, Chicago, 1968. MR 38 \#2765.

4. R. J. Koch, Arcs in partially ordered spaces, Pacific J. Math. 9 (1959), 723-728. MR 21 \#7269.

5. A. D. Wallace, The map excision theorem, Duke Math. J. 19 (1952), 177-182. MR 13, 765.

6. - A theorem on acyclicity, Bull. Amer. Math. Soc. 67 (1961), 123-124. MR 23 \#A2196.

7. - Acyclicity of compact connected semigroups, Fund. Math. 50 (1961/62), 99-105. MR 24 \#A2373.

8. L. E. Ward, Jr., Partially ordered topological spaces, Proc. Amer. Math. Soc. 5 (1954), 144-161. MR 16, 59.

9. - Concerning Koch's theorem on the existence of arcs, Pacific J. Math. 15 (1965), 347-355. MR 31 \#6206.

UNIVERSITY OF OREGON, EUGENE, OREGON 97403 